\title{
Tracing Method of Quality Issues of Gearbox and Application of Rank Sum Test
}

\author{
Hu Hong \\ School of Mechanical Engineering \\ Jilin Engineering Normal University \\ Changchun, China \\ 799517839@qq.com
}

\author{
Li Lianzhi \\ School of Mechanical Engineering \\ Jilin Engineering Normal University \\ Changchun, China \\ 360730815@qq.com
}

\begin{abstract}
Tracing quality issues of complex automobile gearbox is realized, defective parts and assembly procedures are analyzed and confirmed, analytical method for tracing quality issues is provided and the thinking is illustrated. Furthermore, the tracing model for assembly and quality issues of the gearbox is constructed based on inference principles of the rank sum test; the research results show that the analytical method is effective and feasible in tracing quality issues of the gearbox.
\end{abstract}

Keywords- Gearbox; Tracing Method; Quality Issues; Rank Sum Test; Application

\section{INTRODUCTION}

In consideration of an automobile gearbox factory, two questions need settling for tracking quality issues of the gearbox in the running-in test bed; first, there is complex assembly spot, abundant positions and personnel; and tracing analytical thought with strong logicality is needed; second, collected data is discontinuous in time due to shift of operators but strong and irregular in space, so objective inference principles are essential [1]. There are still few papers and references about gearbox tracing method at home and abroad. Thus, we try to propose a tracking idea for a gear factory and built the tracing model by the theory of rank sum test so as to further provide reference value for tracking analysis.

\section{ASSEMBLY PROCESS OF GEARBOX QUALITY PROBLEM PARTS}

The quality of the gearbox is described using a questionnaire. Questionnaire is one of the most common methods of processing quality management data. In the specific application, we can use different formats according to the investigation items and quality characteristics [2]. Table 1 is Questionnaire to integrate the original data from the test bench and the original data in the workshop. The original data of the test bench is a record of the varieties and problems described by the test personnel [3]. Processing method of problem parts be completed by maintenance personnel of the repair shop. From table 1, it can be seen that the main problem of the quality of the gearbox is Qiaoxiang. Qiaoxiang is a regular noise caused by gear surface scar marks in gearbox. The idlers is the main parts caused Qiaoxiang.

The purposes of the quality problem traceability of automobile transmission are: Firstly, which are the main stations making the idler bump in the assembly workshop, Secondly which staff makes the problem parts?

TABLE I. GEARBOX QUALITY PROBLEM QUESTIONNAIRE

\begin{tabular}{|c|c|c|c|}
\hline Quality Problem & Occurrence Frequency & Quality Problem & Occurrence Frequency \\
\hline Qiaoxiang & 72 & Shifting Fork Sound & 3 \\
\hline Shift Difficulty & 30 & Bearing Burning & 1 \\
\hline Neutral & 22 & Side Light don't Lit & 1 \\
\hline Guide Block Problem & 14 & Matching Tight & 1 \\
\hline Abnormal Sound & 7 & Side Light Lit & 1 \\
\hline Shift & 4 & Gorm Motion & 1 \\
\hline Switch Problem & 4 & Gear Position Selection & 1 \\
\hline Gear Tight & 4 & Idling & 1 \\
\hline
\end{tabular}


Before the idler parts into the assembly shop, we assume that there are no quality problems, and then in the assembly line, all the contact station may cause the idler bump. By looking up the process documentation and onsite verification, we knew that the idler assembled into the gearbox housing when into the assembly line would go through three stations, and the relationship between them is connected in series as shown in Fig.1. For the plant, in order to balance assembly work of the assembly line, two test-beds were arranged at the detection station, that is to configure two staff who can detect parts synchronously, and the two detectors in parallel; two transport upper lines at Transport station, that is to configure two transport staff who can transport parts synchronously, and two transport lines in parallel; and three bench configured at assembly station, that is to configure three assembly staff, and the relationship between the three benches is in parallel[4]. Parts firstly pass by testing stations. The detecting staff should carry out detecting for each part. In general case, the profile of idler was detected by the standard gear, the part detected was transported to assembly station workbench by the transport staff of transport station by the means of transport tools. The staff can complete the last installation of idler into the gearbox housing.

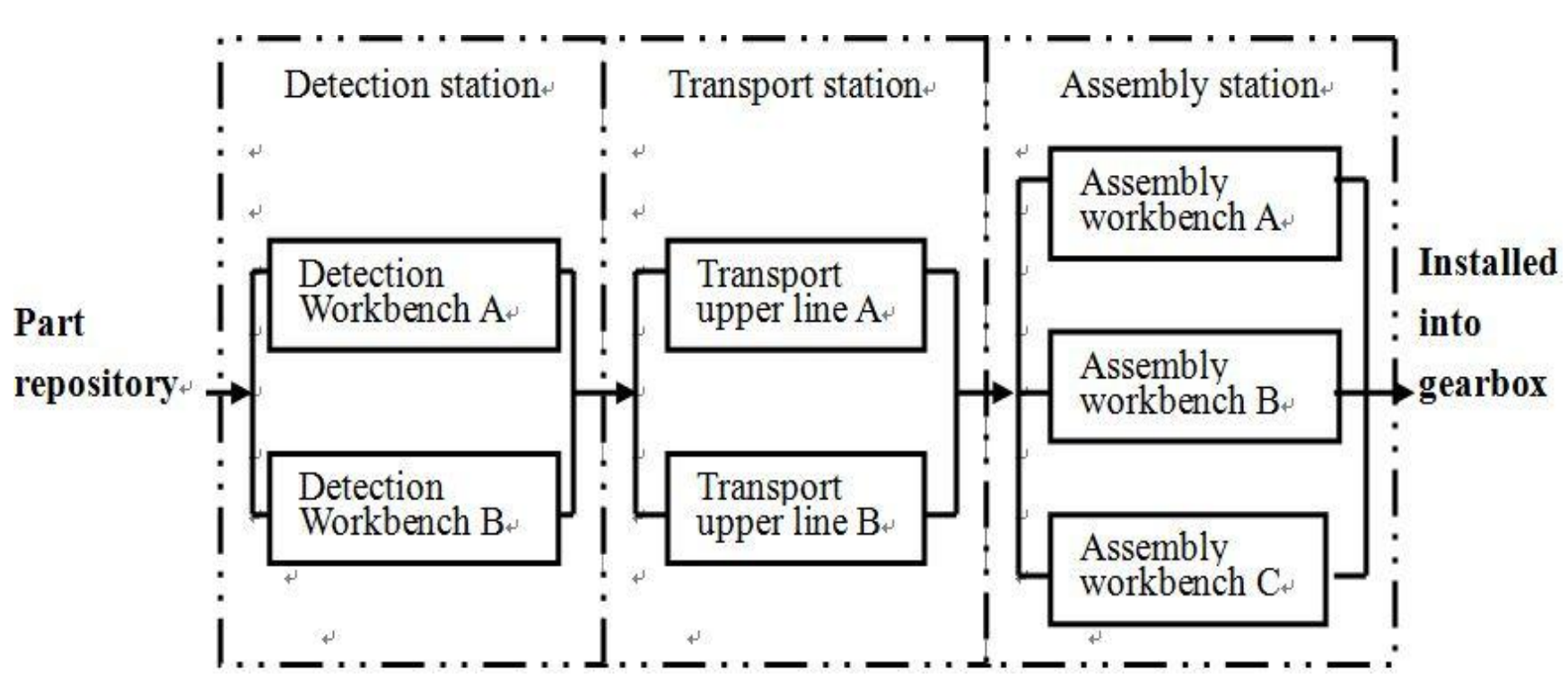

Figure 1. Assembly Processes of Idlers in the Assembly Shop

\section{RESEARCH ON TRACKING METHOD OF ASSEMBLY QUALITY TRACKING OF GEARBOX}

We use reverse tracing method for quality issues of idler parts which aims to issues more directly and discovers where defective parts exist according to reasons of problems [5]. Our tracing ideas are:

(1) Three benches and three workers of the assembly position assemble the idler randomly. Dumped idlers between three benches are distributed similarly, namely the number of dumped idlers on each bench is basically equal. First, there are enough assembly idlers; second, reasons influencing the quality of three benches are almost identical; last, the number of idlers assembled by three workers is identical. If the last condition cannot be met, the probability that defective parts occur in each bench (the ratio $\mathrm{Pi}$ of the number of the bumped idler and the assembly number of the bench) should be consistency when the following conditions are met, namely $P_{A} \approx P_{B} \approx P_{C}$. The same is true with the detection bench and the transport position
(2) If the occurrence rate of defective parts between three assembly benches in the assembly position is rather different and the assembly position with higher occurrence rate is the doubtful, the situation that the bench influences the assembly quality. The same is true with the detection bench and the transport position.

(3) Compare the occurrence rate of two detection benches in the detection position, two transport onlines in the transport position and defective parts in three assembly benches in the assembly position; if the occurrence rate is different, rank the position based on the difference. The higher the occurrence rate is, the more possible the part is defective, namely defective position [6].

(4) Compare the occurrence rate of two detection benches in the detection position, two transport onlines in the transport position and defective parts in three assembly benches in the assembly position; if the occurrence rate is not much different and there are quality issues, we can certainly judge all positions may go wrong. Such special situation is also investigated and studied. If it really happens in a month in light of the assembly site of the gearbox but there are few quality issues in the very month, 
tracing method is proposed in the paper that: quality error corrective measures are designed from the position contacting to the parts first when human factors are eliminated; reduce the occurrence rate of defective parts contacting to the position first down and keep observing.

\section{TRACING OF ASSEMBLY QUALITY ISSUES OF THE GEARBOX BASED ON RACK SUM TEST}

In the assembly quality tracking of the gearbox, collection of data is stereoscopic and different in both time and the number of workers [7]. For example, the number of detectors is different if the number of gearboxes assembled every day is different. A detector can assemble all gearboxes when fewer gearboxes are needed and the other can take a shift. For another example, data of three assemblers is discontinuous in time; if more gearboxes are needed on one day, three assemblers need to work; otherwise, they can be on a shift to balance the assembly line.

We find from quality issues of a gearbox factory that assemblers work in different time, data is generally interlaced and three-dimensional; while such discontinuous data is quite suitable for the rank sum test which is not influenced by the value of data but can reduce the error; moreover, the rank sum rest only cares the rank of the value. It makes no difference between taking rank as the detection target and abnormal data; even though abnormal data changes greatly, it is ranked with a gap from the previous value.

Then, observe and analyze data samples. Although they are distributed uncertainly, the rank sum test is extremely accurate for detecting them with high reliability. The rank sum test is used in the paper based on true and objective original data, enhancing creditability of tracing quality issues of the gearbox [8].

Before the rank sum test, we make statements as follows.

(1) The occurrence rate of defects in a month is detected; there are 7 groups with 7 samples, namely 7 lines of the defect rate. The occurrence rate is the ratio between the number of defective parts and the number of assembly parts.

(2) Only 6 factors on the operation desk influence the quality: man, machine, material, method, environment and detection; generally, workers take place of these factors in the assembly shop.

(3) Each worker contact with parts randomly in the operation. When factors replaced by workers are identical, the occurrence rate should be distributed randomly; otherwise, they are different; namely there are defective workers corresponding to defective positions [9].

The tracing model of the rank sum test can be seen in Fig. 2 which shows tracing processes.
(1) Conduct multi-sample rank sum test to 7 groups of data in three positions, mix 7 all samples values together, calculate the rank of each sample value and detect whether 7 groups belong to an entity. If the result shows that 7 groups do not belong to an entity, some out of 7 workers come across defective parts, but which worker comes across quality issues should be eliminated one by one.

(2) First, eliminate the first detection position of contacted parts and the defective occurrence rate of two workers is two samples. Conduct rank sum test to them. If two samples belong to an entity, eliminate the detection position; otherwise, the detection position is defective.

(3) Then, eliminate the second transport position of contacted parts and the defective occurrence rate of two workers is two samples. Conduct rank sum test to them. If two samples belong to an entity, eliminate the transport position; otherwise, the transport position is defective.

(4) Finally, eliminate the last position of contacted parts and the defective occurrence rate of three workers is three samples. Conduct rank sum test to them. If three samples belong to an entity, eliminate the assembly position; otherwise, the assembly position is defective.

(5) When multi-sample rank sum test is conducted to 7 samples of three positions and the defective occurrence rate of workers is distributed in an entity randomly, it means that the occurrence rate is identical. Since there are defective parts on the spot, we can doubt that all workers come across defective parts. Besides, we have also investigated and studied such special situation. It can be seen from the assembly site that if such situation occurs in a month and quality issues are rare, tracing method is proposed in the paper that: quality error corrective measures are designed from the position contacting to the parts first when human factors are eliminated; reduce the occurrence rate of defective parts contacting to the position first down and keep observing [10].

(6) After the rank sum test, analyze quality issues of the gearbox, confirm major defective positions and workers and propose improvement suggestions. Tracing Model of Rank Sum Test is shown as Fig.2.

\section{CONCLUSION}

It is unconvincing to diagnose quality issues of a gearbox factory based on previous experience. In the research, we make a conclusion that the tracing method is pointed and practical with the assembly site under coordination of the assembly shop; a tracing method based on rank sum test and in accordance with collected data is proposed; in particular, a tracing model based on rank sum test is built which makes the tracing process clearer for front-line workers, laying objective foundation for tracing results and providing forceful supports for changing data information into decision information. 


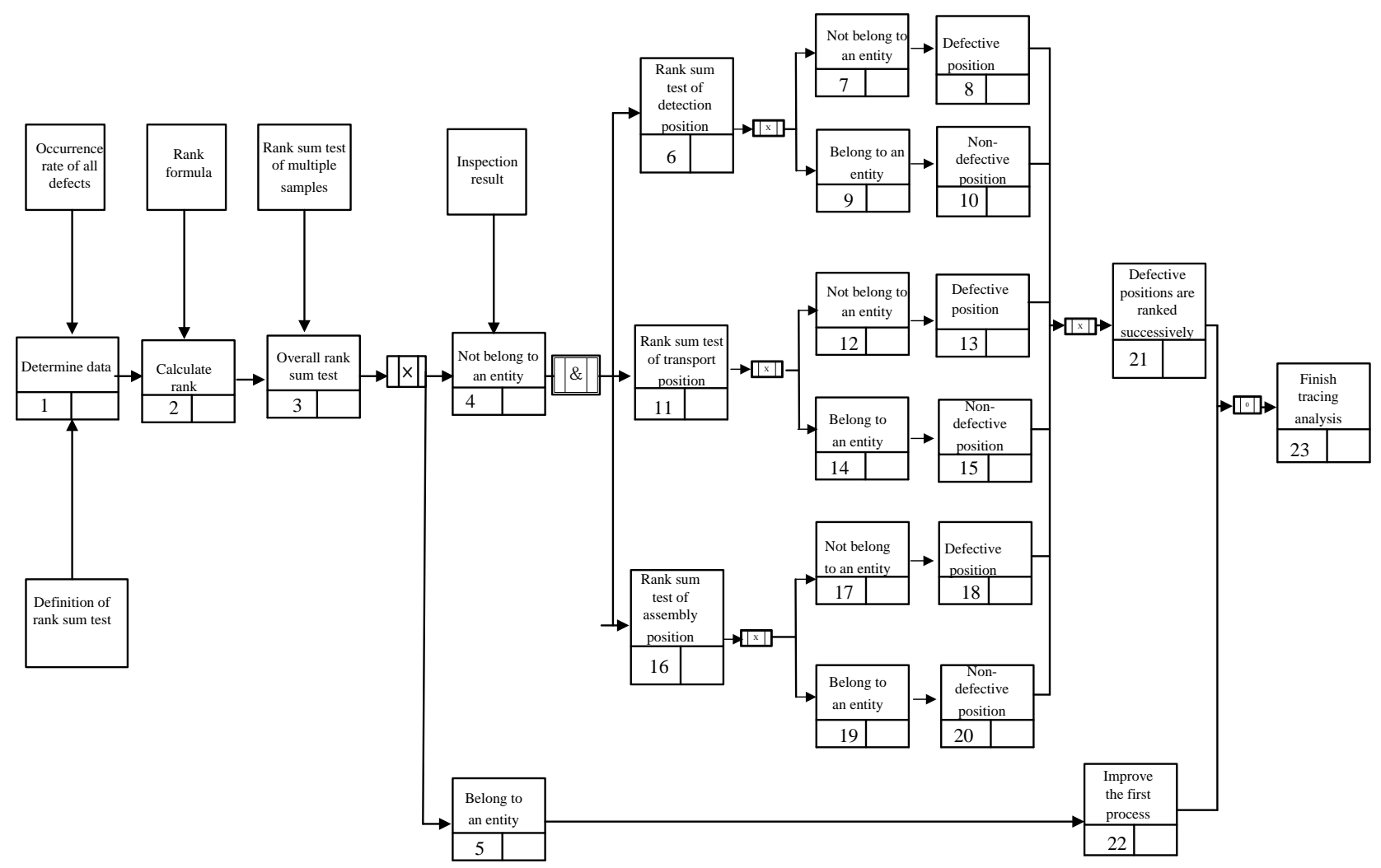

Figure 2. Tracing Model of Rank Sum Test

\section{REFERENCES}

[1] Tan Xin, You Jianxin, Shao Runing, "Quality management theories and methods," Dongbei University of Finance and Economics Press, 2009.

[2] Mao Shisong, Zhou Jixiang, "Statistical methods for quality management," China Statistics Press, 2011.

[3] Valia T, Petkova P C S A, "The use of quality metrics inservice centres,'Int.J.Production Economics, 2000(7):29-31.

[4] Atto A M, Pastor D, Mercier G, "Detection threshold for nonparametric estimation," Signal,Image and Video Processing, 2008,2(3):207-223.

[5] Bannayan M, Hoogenboom G, "Predicting realizations of daily weather data for climate forecasts using the non-parametric nearest-neighbour re-sampling technique," International Journal of Climatology, 2008,28(10):1357-1368.

[6] Choi W, Lee J W, Huh M H, et al, "An Algorithm for Computing the Exact Distribution of the Kruskal-Wallis Test," Communications in StatisticsPartB:Simulationand Computation, 2003,32(4): 1029-1040.

[7] ]Combe Nencka H, Combe P, "Riemannian geometry on nonparametric probability space," Neural Network World, 2006,16(6):459-473.

[8] DAS N, "A comparison study of three non-parametric control charts to detect shift in location parameters," International Journal of Advanced Manufacturing Technology, 2009,41(7-8):799-807.

[9] JERRY LANDT T, "Shrouds of Time The history of RFID," An AIM Publication, 2001:56-58.

[10] LAZARO A, GIRBAUD, VILLARINOR,"Effects of interferences in UHF RFID Systems ," Progress in Electromagnetics Research, 2009, 98:425-443. 